\title{
Performance of Stochastically Intermittent Sensors in Detecting a Target Traveling between Two Areas
}

\author{
Hongyun Wang ${ }^{*}$, Hong Zhou ${ }^{2 *}$ \\ ${ }^{1}$ Department of Applied Mathematics and Statistics, Baskin School of Engineering, University of California, \\ Santa Cruz, CA, USA \\ ${ }^{2}$ Department of Applied Mathematics, Naval Postgraduate School, Monterey, CA, USA \\ Email: "hongwang@soe.ucsc.edu, "hzhou@nps.edu
}

Received 6 November 2015; accepted 11 March 2016; published 15 March 2016

Copyright (C) 2016 by authors and Scientific Research Publishing Inc.

This work is licensed under the Creative Commons Attribution International License (CC BY). http://creativecommons.org/licenses/by/4.0/

(c)

\begin{abstract}
We study the problem of detecting a target that moves between a hiding area and an operating area over multiple fixed routes. The research is carried out with one or more cookie-cutter sensors with stochastic intermission, which turn on and off stochastically governed by an on-rate and an off-rate. A cookie-cutter sensor, when it is on, can detect the target instantly once the target comes within the detection radius of the sensor. In the hiding area, the target is shielded from being detected. The residence times of the target, respectively, in the hiding area and in the operating area, are exponentially distributed and are governed by rates of transitions between the two areas. On each travel between the two areas and in each travel direction, the target selects a route randomly according to a probability distribution. Previously, we analyzed the simple case where the sensors have no intermission (i.e., they stay on all the time). In the current study, the sensors are stochastically intermittent and are synchronized (i.e., they turn on or off simultaneously). This happens when all sensors are affected by the same environmental factors. We derive asymptotic expansions for the mean time to detection when the on-rate and off-rate of the sensors are large in comparison with the rates of the target traveling between the two areas. Based on the mean time to detection, we evaluate the performance of placing the sensor(s) to monitor various travel route(s) or to scan the operating area.
\end{abstract}

\section{Keywords}

Stochastically Intermittent Sensors, Moving Target with Constrained Pathways, Mean Time to Detection, Optimal Search Design

\footnotetext{
${ }^{*}$ Corresponding authors.

How to cite this paper: Wang, H.Y. and Zhou, H. (2016) Performance of Stochastically Intermittent Sensors in Detecting a Target Traveling between Two Areas. American Journal of Operations Research, 6, 199-212. 


\section{Introduction}

Search and detection theory has a history of principal importance in operations research. It has fundamental military and civilian applications such as anti-submarine warfare, counter-mine warfare, and search and rescue operations [1]-[4].

Nowadays when combating piracy at sea, detecting and intercepting threat objects (boats) such as terrorists and drug or weapon smugglers, or securing coastlines and trade routes, it is important to understand the behavior of a target and plan a search and detection strategy accordingly. In a recent work [5] we considered the problem of searching for a target that travels between a hiding area and an operating area via multiple routes. By assuming certain behaviors of the moving target, we obtained analytic expressions for the mean time to detection and thereby were able to determine the optimal placement of $m$ cookie-cutter sensors (i.e. how many sensors should we place on which routes and the rest is placed to search the operating area). Interestingly, we found that the optimal placement, sometimes, is not the one suggested by intuition.

In this paper we would like to extend our earlier study to include stochastically intermittent sensors in detecting a target moving between a hiding area and an operating area.

\section{Mathematical Formulation for the Case of Sensors without Intermission}

We consider the search problem in which a target moves between a hiding area and an operating area via constrained pathways, as depicted in Figure 1. The target can stay in the hiding area where the target is shielded from being detected by the sensors. There are $N$ given routes connecting the hiding area and the operating area. The target can travel along one of these $N$ given routes from the hiding area to the operating area. The target can spend time in the operating area to carry out certain activities/tasks. Afterwards, the target can return to the hiding area via possibly a different route. In the hiding area, the target is not detectable. Outside the hiding area, the target is detectable along the routes and in the operating area if it comes into the detection range of a sensor.

In the search problem, variable number of synchronized intermittent cookie-cutter sensors are used to detect the target. We will first introduce the mathematical model for the simple case of non-intermittent sensors (i.e., they stay on all the time) and then extend the model to accommodate the stochastic intermission of the sensors.

We start by specifying the target behavior. The target moves stochastically between the hiding area and the operating area according to the following rules.

- The dwell time of the target in the hiding area is exponentially distributed with rate $r_{f}$, the forward rate of the target going from the hiding area to the operating area.

- On its travel from the hiding area to the operating area, the target takes route $k$ with probability $p_{k}$, which satisfies the constraint $\sum_{k=1}^{N} p_{k}=1$ where $N$ is the total number of given routes.

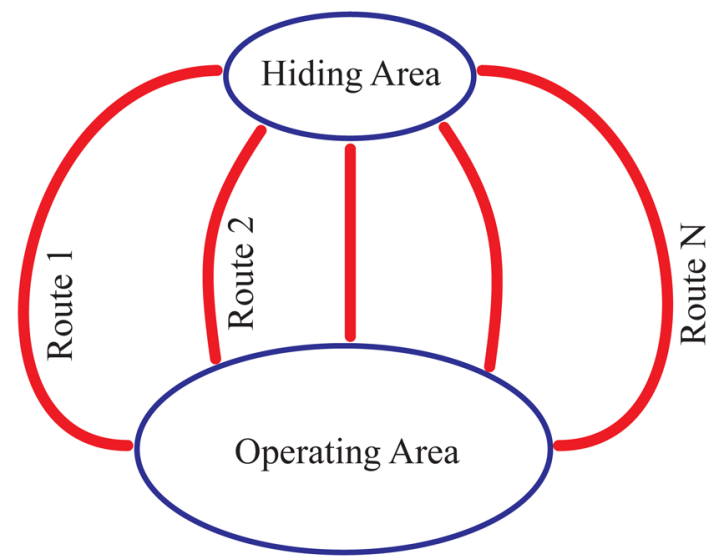

Figure 1. A target traveling between two areas. The target may stay in the hiding area where it is not detectable; it may travel from the hiding area to the operating area along one of the $N$ given routes; it may spend time in the operating area before returning to the hiding area via possibly a different route. 
- The dwell time of the target in the operating area is exponentially distributed with rate $r_{b}$, the backward rate of the target going from the operating area back to the hiding area.

- On its travel from the operating area back to the hiding area, the target chooses route $k$ with probability $q_{k}$, which satisfies the condition $\sum_{k=1}^{N} q_{k}=1$.

- The travel time between the operating area and the hiding area is negligible in comparison with the dwell times in the hiding area and the operating area. Mathematically, we treat the travel time along a route as zero.

The target's travel between the two areas is mathematically described by a Markov process of two states, with forward rate $r_{f}$ and backward rate $r_{b}$, as illustrated in Figure 2 .

Next, we describe the interaction between the target and sensors. A non-intermittent cookie-cutter sensor is an ideal sensor which detects the target instantly once the target comes within distance $R$ to the center of the sensor where the radius $R$ is called the detection radius of the sensor. When the target is outside the detection radius, it is not detected. In this study, we assume that the detection radius of sensors is large enough to cover the full width of any one of the given routes. Consequently, if a non-intermittent sensor is assigned to monitor a route and the target happens to move along that route, the target will definitely be detected by the sensor. Of course, the situation will be different for an intermittent sensor that turns on and off stochastically.

When a sensor is used to search the operating area, when the sensor is on, and when the target is in the operating area, the interaction between the target and the sensor is modeled using a detection rate $r_{d}$ (probability of detection per time).

$$
r_{d} \equiv \text { rate of detecting the target in the operating area }
$$

This detection rate is affected by the size of the operating area, and by the detection radius and the speed of the sensor.

When one or more non-intermittent sensors are deployed to monitor one or more routes or to search the operating area, the transitions and detection of the target are governed by a 3-state Markov process of the same parameter form as the one shown in Figure 3. The values of $p, q$, and $d$ depend on how many sensors are used and which route(s) and area are monitored/searched. Values of $p, q$, and $d$ are given below for several cases.

- When only one sensor is deployed and it is placed to monitor route $k$, this gives

$$
p=p_{k}, \quad q=q_{k}, \quad d=0
$$

- When only one sensor is deployed and it is used to search the operating area, this corresponds to

$$
p=0, \quad q=0, \quad d=r_{d}
$$

- When two sensors are placed to monitor, respectively, routes $k$ and $j(k \neq j)$, it follows that

$$
p=p_{k}+p_{j}, \quad q=q_{k}+q_{j}, \quad d=0
$$

- When two sensors are both used to search the operating area, we find

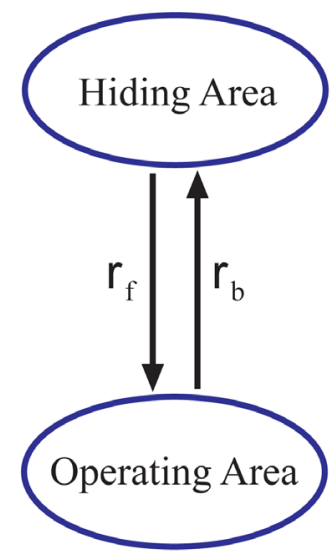

Figure 2. Markov transitions of the target between the hiding area and the operating area in the absence of any sensor. 


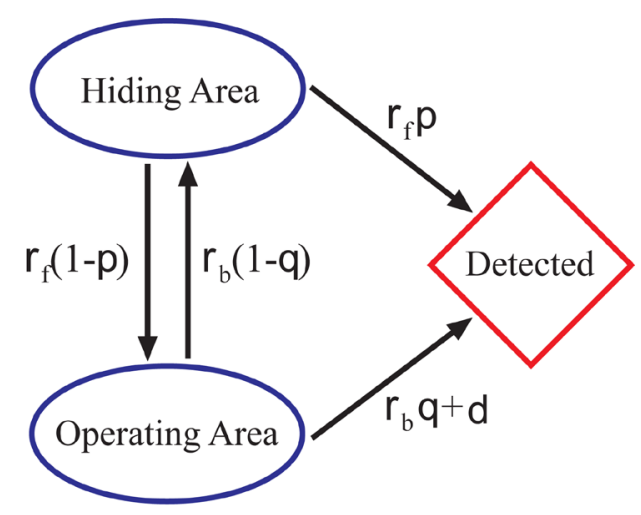

Figure 3. Markov transitions of the target in the presence of non-intermittent sensor(s). Note that the parameter form of the Markov process is the same for all cases while values of parameter $p, q$, and $d$ vary from case to case.

$$
p=0, \quad q=0, \quad d=2 r_{d}
$$

- When one sensor is placed to monitor route $k$ and a second sensor is used to search the operating area, this gives

$$
p=p_{k}, \quad q=q_{k}, \quad d=r_{d}
$$

To facilitate our analysis, we divide all transition rates by the sum $\left(r_{f}+r_{b}\right)$ to non-dimensionalize the Markov process depicted in Figure 3. Additionally, we introduce two dimensionless parameters:

$$
\alpha \equiv \frac{r_{f}}{r_{f}+r_{b}}, \quad \mu \equiv \frac{d}{r_{f}+r_{b}}
$$

The parameter form of the normalized Markov process is shown in Figure 4.

In the absence of sensors, at equilibrium, the probabilities of the target being in the hiding area or the operating area are, respectively, $(1-\alpha)$ and $\alpha$. This fact will be used later in the calculation of mean time to detection.

\section{Mean Time to Detection in the Case of Sensors without Intermission}

Figure 4 describes the general model for the case of non-intermittent sensors. It can accommodate arbitrary number of sensors. For mathematical convenience, we label the hiding area as state 1 and the operating area as state 2 . We solve for the mean time to detection.

Let $T$ denote the time to detection (random variable), and $S(t)$ denote the state of the target at time $t$. Time 0 is defined as the time when the sensors are deployed. We examine the conditional mean time to detection given that the target is in state $j$ at time 0 .

$$
t_{j} \equiv E(T \mid S(0)=j), \quad j=1,2
$$

We derive two equations for $t_{1}$ and $t_{2}$ based on the Markov process in Figure 4. Starting with $S(0)=1$, the probability distribution of $S(\Delta t)$ is

$$
S(\Delta t)= \begin{cases}1, & \text { with probability } 1-\alpha \Delta t+o(\Delta t) \\ 2, & \text { with probability } \alpha(1-p) \Delta t+o(\Delta t) \\ \text { detected, } & \text { with probability } \alpha p \Delta t+o(\Delta t)\end{cases}
$$

Using the law of total expectation, we have

$$
t_{1}=\Delta t+(1-\alpha \Delta t) t_{1}+\alpha(1-p) \Delta t \cdot t_{2}+o(\Delta t)
$$




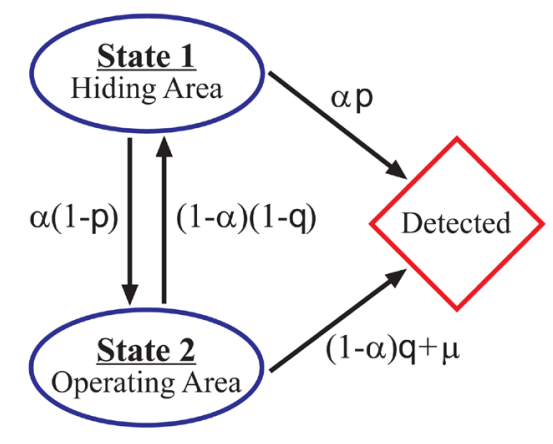

Figure 4. The normalized Markov process governing the transitions of the target.

which, when divided by $\Delta t$ and in the limit of $\Delta t$ going to zero, yields

$$
1-\alpha t_{1}+\alpha(1-p) t_{2}=0
$$

This is an equation for $t_{1}$ and $t_{2}$. Similarly, another equation can be derived starting with $S(0)=2$. The two equations for $t_{1}$ and $t_{2}$ are

$$
\left\{\begin{array}{l}
1-\alpha t_{1}+\alpha(1-p) t_{2}=0 \\
1-((1-\alpha)+\mu) t_{2}+(1-\alpha)(1-q) t_{1}=0
\end{array}\right.
$$

Solving linear system (5), we obtain

$$
\left\{\begin{array}{l}
t_{1}=\frac{\frac{\mu}{\alpha(1-\alpha)}+\frac{1-p}{1-\alpha}+\frac{1}{\alpha}}{\frac{\mu}{1-\alpha}+p+q-p q} \\
t_{2}=\frac{\frac{1-q}{\alpha}+\frac{1}{1-\alpha}}{\frac{\mu}{1-\alpha}+p+q-p q}
\end{array}\right.
$$

Before the deployment of sensors, the equilibrium distribution of the target is

$$
\operatorname{Pr}(S(0)=1)=1-\alpha, \quad \operatorname{Pr}(S(0)=2)=\alpha
$$

Thus, the overall mean time to detection has the expression

$$
E(T)=(1-\alpha) t_{1}+\alpha t_{2}=\frac{\frac{\mu}{\alpha}-(p+q)+\frac{1}{\alpha(1-\alpha)}}{\frac{\mu}{1-\alpha}+p+q-p q}
$$

\section{Mathematical Formulation for the Case of Synchronized Stochastically Intermittent Sensors}

We extend above discussions to consider synchronized intermittent sensors that stochastically turn on or off simultaneously. We model the stochastic evolution of sensors as a 2-state Markov process with an on-rate $r_{\text {on }}$ and an off-rate $r_{\text {off }}$, as illustrated in Figure 5.

As in the previous section, we divide all rates by $\left(r_{f}+r_{b}\right)$ to normalize the problem. After the nondimensionalization scaling, we write the on-rate and the off-rate as

$$
\text { on-rate }=\frac{r_{\text {on }}}{r_{f}+r_{b}}=\frac{\beta}{\varepsilon}
$$




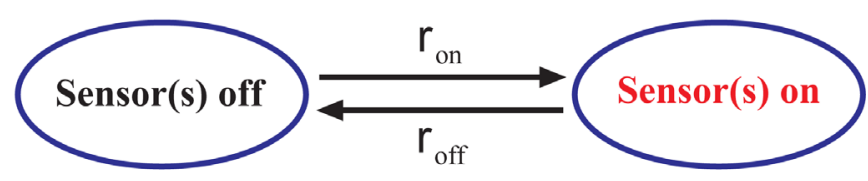

(a) Transitions of the sensor(s) before scaling

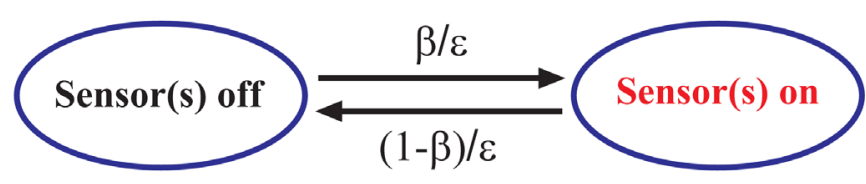

(b) Transitions of the sensor(s) after scaling

Figure 5. The 2-state Markov process governing the intermittent sensors.

$$
\text { off-rate }=\frac{r_{\text {off }}}{r_{f}+r_{b}}=\frac{1-\beta}{\varepsilon}
$$

where

$$
\varepsilon=\frac{r_{f}+r_{b}}{r_{\mathrm{on}}+r_{\mathrm{off}}}, \quad \beta=\frac{r_{\mathrm{on}}}{r_{\mathrm{on}}+r_{\mathrm{off}}}
$$

Note that the parameter $\beta$ defined above corresponds to the equilibrium probability of sensors being on, which is also the fraction of time that sensors are on. The quantity $(1-\beta)$ is the equilibrium probability of sensors being off. The choice of notation $\varepsilon$ indicates that we will focus on the parameter regime of $\varepsilon \ll 1$. That is, we will focus on the case of $\left(r_{\text {on }}+r_{\text {off }}\right) \gg\left(r_{f}+r_{b}\right)$. Note that $\left(r_{\text {on }}+r_{\text {off }}\right)$ is the rate of sensors relaxing to equilibrium between the on- and off-states; $\left(r_{f}+r_{b}\right)$ is the rate of the target relaxing to equilibrium between the hiding area and the operating area. Parameter $\varepsilon$ in (9) measures the ratio of these two relaxation rates.

We now combine the stochastic travel of the target and the stochastic intermission of the sensors into a 5-state Markov process for the target-sensors system as illustrated in Figure 6. The normalized version of this 5-state Markov process where all transition rates are divided by $\left(r_{f}+r_{b}\right)$, is shown in Figure 7 . In the model, the sensors' intermission and the target's travel between the two areas are independent of each other. The targetsensors system can reside in any of 4 states before the detection. For mathematical convenience, we number the 4 states as follows: (Figure 7).

- State 1: the target is in the hiding area and the sensors are on.

- State 2: the target is in the operating area and the sensors are on.

- State 3: the target is in the hiding area and the sensors are off.

- State 4: the target is in the operating area and the sensors are off.

Figure 7 gives the general model for the target-sensors system with synchronized stochastically intermittent sensors. It can accommodate arbitrary number of sensors. We study the mean time to detection in this 5-state Markov process.

Again, let $T$ represent the time to detection (random variable), and $S(t)$ denote the state of the target at time $t$. We consider the conditional mean time to detection

$$
t_{j} \equiv E(T \mid S(0)=j), \quad j=1,2,3,4
$$

We derive four equations for $\left(t_{1}, t_{2}, t_{3}, t_{4}\right)$ based on the 5-state Markov process in Figure 7. Starting with $S(0)=1$, the probability distribution of $S(\Delta t)$ is 


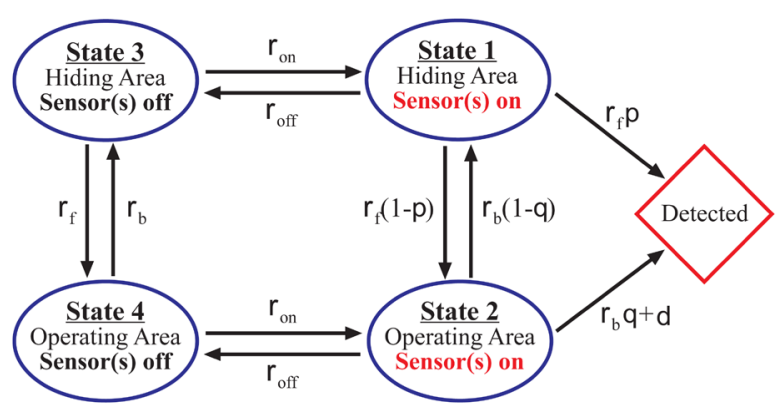

Figure 6. The 5-state Markov process governing the targetsensors system.

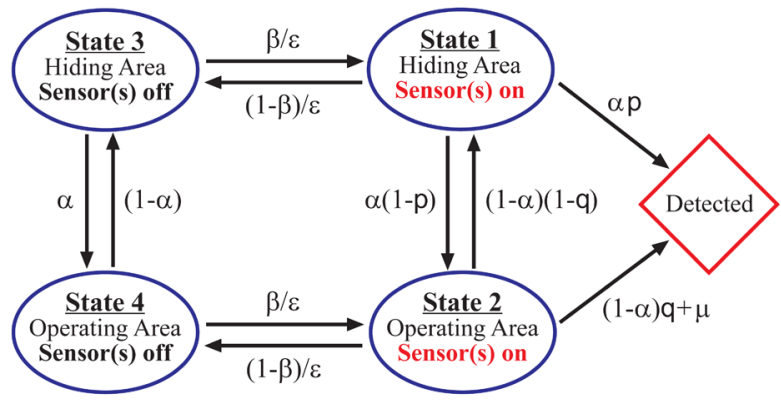

Figure 7. The normalized 5-state Markov process governing the target-sensors system. In the non-dimensionalization scaling, all transition rates are divided by $\left(r_{f}+r_{b}\right)$.

$$
S(\Delta t)= \begin{cases}1, & \text { with probability } 1-\left(\alpha+\frac{1-\beta}{\varepsilon}\right) \Delta t+o(\Delta t) \\ 2, & \text { with probability } \alpha(1-p) \Delta t+o(\Delta t) \\ 3, & \text { with probability } \frac{1-\beta}{\varepsilon} \Delta t+o(\Delta t) \\ \text { detected, } & \text { with probability } \alpha p \Delta t+o(\Delta t)\end{cases}
$$

The law of total expectation gives us

$$
t_{1}=\Delta t+\left(1-\left(\alpha+\frac{1-\beta}{\varepsilon}\right) \Delta t\right) t_{1}+\alpha(1-p) \Delta t \cdot t_{2}+\frac{1-\beta}{\varepsilon} \Delta t \cdot t_{3}+o(\Delta t)
$$

Dividing both sides by $\Delta t$ and taking the limit as $\Delta t$ going to zero, we arrive at

$$
1-\left(\alpha+\frac{1-\beta}{\varepsilon}\right) t_{1}+\alpha(1-p) t_{2}+\frac{1-\beta}{\varepsilon} t_{3}=0
$$

This is an equation for $\left(t_{1}, t_{2}, t_{3}, t_{4}\right)$. Three other equations are derived using the same approach, starting with, respectively, $S(0)=2, S(0)=3$, and $S(0)=4$. Putting together, these four equations for $\left(t_{1}, t_{2}, t_{3}, t_{4}\right)$ are

$$
\begin{gathered}
1-\left(\alpha+\frac{1-\beta}{\varepsilon}\right) t_{1}+\alpha(1-p) t_{2}+\frac{1-\beta}{\varepsilon} t_{3}=0 \\
1-\left((1-\alpha)+\mu+\frac{1-\beta}{\varepsilon}\right) t_{2}+(1-\alpha)(1-q) t_{1}+\frac{1-\beta}{\varepsilon} t_{4}=0 \\
1-\left(\alpha+\frac{\beta}{\varepsilon}\right) t_{3}+\alpha t_{4}+\frac{\beta}{\varepsilon} t_{1}=0
\end{gathered}
$$




$$
1-\left((1-\alpha)+\frac{\beta}{\varepsilon}\right) t_{4}+(1-\alpha) t_{3}+\frac{\beta}{\varepsilon} t_{2}=0
$$

Analytical solutions to the above linear system is hard to obtain. Instead, in the next section, we use this linear system to calculate asymptotic expansions for $\left(t_{1}, t_{2}, t_{3}, t_{4}\right)$ and for the overall mean time to detection when $\varepsilon$ is small.

\section{Asymptotic Solutions for the Mean Time to Detection in the Case of Synchronized Intermittent Sensors}

We derive asymptotic solutions for the mean time to detection for small $\varepsilon$. We start by writing the conditional mean time to detection in the asymptotic form of

$$
t_{j}=t_{j}^{(0)}+\varepsilon t_{j}^{(1)}+\cdots
$$

Substituting this asymptotic form into Equations (12)-(15) and examining terms of the order $O\left(\frac{1}{\varepsilon}\right)$, which are of the largest magnitude, we obtain

$$
\left\{\begin{array}{l}
t_{1}^{(0)}=t_{3}^{(0)} \\
t_{2}^{(0)}=t_{4}^{(0)}
\end{array}\right.
$$

From Equations (12)-(15), we will derive two equations that do not contain any coefficient of the order $O\left(\frac{1}{\varepsilon}\right)$. These two equations will then be used to calculate the leading coefficients in the asymptotic form: $t_{1}^{(0)}$ and $t_{2}^{(0)}$. We rewrite Equations (12)-(15) to separate terms with coefficients $O\left(\frac{1}{\varepsilon}\right)$ from other terms.

$$
\begin{gathered}
-\frac{1-\beta}{\varepsilon}\left(t_{3}-t_{1}\right)=1-\alpha t_{1}+\alpha(1-p) t_{2} \\
-\frac{1-\beta}{\varepsilon}\left(t_{4}-t_{2}\right)=1-((1-\alpha)+\mu) t_{2}+(1-\alpha)(1-q) t_{1} \\
\frac{\beta}{\varepsilon}\left(t_{3}-t_{1}\right)=1-\alpha t_{3}+\alpha t_{4} \\
\frac{\beta}{\varepsilon}\left(t_{4}-t_{2}\right)=1-(1-\alpha) t_{4}+(1-\alpha) t_{3}
\end{gathered}
$$

The two equations are constructed by

$$
\begin{aligned}
& \beta \times \text { equation }(18)+(1-\beta) \times \text { equation (20) } \\
& \beta \times \text { equation }(19)+(1-\beta) \times \text { equation (21) }
\end{aligned}
$$

The resulting two equations are

$$
\begin{gathered}
1-\beta \alpha t_{1}+\beta \alpha(1-p) t_{2}-(1-\beta) \alpha t_{3}+(1-\beta) \alpha t_{4}=0 \\
1-\beta((1-\alpha)+\mu) t_{2}+\beta(1-\alpha)(1-q) t_{1} \\
-(1-\beta)(1-\alpha) t_{4}+(1-\beta)(1-\alpha) t_{3}=0
\end{gathered}
$$

Substituting asymptotic form (16) into the two equations above, keeping only terms of order $O(1)$, and using result (17), we arrive at

$$
\left\{\begin{array}{l}
\left.1-\alpha t_{1}^{(0)}+\alpha(1-\beta p)\right) t_{2}^{(0)}=0 \\
1-((1-\alpha)+\beta \mu) t_{2}^{(0)}+(1-\alpha)(1-\beta q) t_{1}^{(0)}=0
\end{array}\right.
$$


System (24) is of the same parameter form as system (5) except that $p, q$, and $\mu$ in (5) are replaced by $\beta p$, $\beta q$, and $\beta \mu$ in (24). The solution of (24) immediately gives us the leading coefficients:

$$
\left\{\begin{array}{l}
t_{1}^{(0)}=t_{3}^{(0)}=\frac{\frac{\beta \mu}{\alpha(1-\alpha)}+\frac{1-\beta p}{1-\alpha}+\frac{1}{\alpha}}{\frac{\beta \mu}{1-\alpha}+\beta p+\beta q-\beta^{2} p q} \\
t_{2}^{(0)}=t_{4}^{(0)}=\frac{\frac{1-\beta q}{\alpha}+\frac{1}{1-\alpha}}{\frac{\beta \mu}{1-\alpha}+\beta p+\beta q-\beta^{2} p q}
\end{array}\right.
$$

We introduce quantity $\sigma \equiv \frac{\alpha(1-\alpha)}{\beta}\left(t_{1}^{(0)}-t_{2}^{(0)}\right)$, which will be useful in the subsequent calculation. Quantity $\sigma$ has the expression

$$
\sigma \equiv \frac{\alpha(1-\alpha)}{\beta}\left(t_{1}^{(0)}-t_{2}^{(0)}\right)=\frac{\mu-\alpha p+(1-\alpha) q}{\frac{\beta \mu}{1-\alpha}+\beta p+\beta q-\beta^{2} p q}
$$

Next, we calculate coefficients $\left(t_{1}^{(1)}, t_{2}^{(1)}, t_{3}^{(1)}, t_{4}^{(1)}\right)$. Substituting the asymptotic form (16) into equation (20)(21), and using the fact that all leading coefficients are already known, we find expressions for $\left(t_{3}^{(1)}-t_{1}^{(1)}\right)$ and $\left(t_{4}^{(1)}-t_{2}^{(1)}\right)$.

$$
\begin{gathered}
t_{3}^{(1)}-t_{1}^{(1)}=\frac{1}{\beta}\left(1-\alpha t_{1}^{(0)}+\alpha t_{2}^{(0)}\right)=\frac{1}{\beta}-\frac{\sigma}{1-\alpha} \\
t_{4}^{(1)}-t_{2}^{(1)}=\frac{1}{\beta}\left(1-(1-\alpha) t_{2}^{(0)}+(1-\alpha) t_{1}^{(0)}\right)=\frac{1}{\beta}+\frac{\sigma}{\alpha} \\
\left(t_{4}^{(1)}-t_{2}^{(1)}\right)-\left(t_{3}^{(1)}-t_{1}^{(1)}\right)=\frac{\sigma}{\alpha(1-\alpha)}
\end{gathered}
$$

Substituting the asymptotic form (16) into Equations (22)-(23), collecting all terms of the order $O(\varepsilon)$, and using results (27)-(28), we obtain two equations for $t_{1}^{(1)}$ and $t_{2}^{(1)}$.

$$
\left\{\begin{array}{l}
t_{1}^{(1)}-(1-\beta p) t_{2}^{(1)}=\frac{1-\beta}{\alpha(1-\alpha)} \sigma \\
(1-\beta q) t_{1}^{(1)}-\left(1+\frac{\beta \mu}{1-\alpha}\right) t_{2}^{(1)}=\frac{1-\beta}{\alpha(1-\alpha)} \sigma
\end{array}\right.
$$

The solution of (30) gives us expressions for coefficients $\left(t_{1}^{(1)}, t_{2}^{(1)}, t_{3}^{(1)}, t_{4}^{(1)}\right)$.

$$
\left\{\begin{array}{l}
t_{1}^{(1)}=\frac{\frac{\beta \mu}{1-\alpha}+\beta p}{\frac{\beta \mu}{1-\alpha}+\beta p+\beta q-\beta^{2} p q} \cdot \frac{1-\beta}{\alpha(1-\alpha)} \sigma \\
t_{2}^{(1)}=\frac{-\beta q}{\frac{\beta \mu}{1-\alpha}+\beta p+\beta q-\beta^{2} p q} \cdot \frac{1-\beta}{\alpha(1-\alpha)} \sigma \\
t_{3}^{(1)}=t_{1}^{(1)}+\left(\frac{1}{\beta}-\frac{\sigma}{1-\alpha}\right) \\
t_{4}^{(1)}=t_{2}^{(1)}+\left(\frac{1}{\beta}+\frac{\sigma}{\alpha}\right)
\end{array}\right.
$$

Before the sensors are assigned to monitor/search routes, the equilibrium distribution of the target-sensors 
system is

$$
\begin{array}{cc}
\operatorname{Pr}(S(0)=1)=(1-\alpha) \beta, & \operatorname{Pr}(S(0)=2)=\alpha \beta \\
\operatorname{Pr}(S(0)=3)=(1-\alpha)(1-\beta), & \operatorname{Pr}(S(0)=4)=\alpha(1-\beta)
\end{array}
$$

It follows that the overall mean time to detection has the expression

$$
\begin{aligned}
E(T)= & (1-\alpha) \beta t_{1}+\alpha \beta t_{2}+(1-\alpha)(1-\beta) t_{3}+\alpha(1-\beta) t_{4} \\
= & \left((1-\alpha) \beta t_{1}^{(0)}+\alpha \beta t_{2}^{(0)}+(1-\alpha)(1-\beta) t_{3}^{(0)}+\alpha(1-\beta) t_{4}^{(0)}\right) \\
& +\varepsilon\left((1-\alpha) \beta t_{1}^{(1)}+\alpha \beta t_{2}^{(1)}+(1-\alpha)(1-\beta) t_{3}^{(1)}+\alpha(1-\beta) t_{4}^{(1)}\right)
\end{aligned}
$$

Therefore, the overall mean time to detection has the asymptotic expansion

$$
E(T)=t^{(0)}+\varepsilon t^{(1)}+\cdots
$$

where the coefficients $t^{(0)}$ and $t^{(1)}$ are given by

$$
\begin{gathered}
t^{(0)}=\frac{\frac{\mu}{\alpha}-p-q+\frac{1}{\beta \alpha(1-\alpha)}}{\frac{\mu}{1-\alpha}+p+q-\beta p q} \\
t^{(1)}=\frac{(\mu+p-\alpha(p+q))(\mu+q-\alpha(p+q))}{\left(\frac{\mu}{1-\alpha}+p+q-\beta p q\right)^{2} \alpha(1-\alpha)} \cdot \frac{(1-\beta)}{\beta}+\frac{1-\beta}{\beta}
\end{gathered}
$$

The normalized transition rates and parameters (shown in Figure 7) are related to the physical transition rates (shown in Figure 6) as follows

$$
\begin{gathered}
\mu \equiv \frac{d}{r_{f}+r_{b}} \\
\alpha \equiv \frac{r_{f}}{r_{f}+r_{b}} \\
\beta \equiv \frac{r_{\text {on }}}{r_{\text {on }}+r_{\text {off }}} \\
\varepsilon \equiv \frac{r_{f}+r_{b}}{r_{\text {on }}+r_{\text {off }}}
\end{gathered}
$$

\section{Behaviors of the Mean Time to Capture}

We examine behaviors of the mean time to detection, $E(T)=t^{(0)}+\varepsilon t^{(1)}$, as a function of parameters $\mu, \alpha$, $\beta, p, q$, and $\varepsilon$.

Observation 1: $t^{(0)}$ is a decreasing function of $\mu$.

We differentiate $t^{(0)}$ as defined in (33) with respect to $\mu$ and find that

$$
\begin{aligned}
\frac{\mathrm{d} t^{(0)}}{\mathrm{d} \mu} & =\frac{\mathrm{d}}{\mathrm{d} \mu}\left(\frac{\frac{\mu}{\alpha}-p-q+\frac{1}{\beta \alpha(1-\alpha)}}{\frac{\mu}{1-\alpha}+p+q-\beta p q}\right) \\
& =-\frac{(1-\beta(1-\alpha) p)(1-\beta(1-\alpha) q)}{\beta \alpha(1-\alpha)^{2}\left(\frac{\mu}{1-\alpha}+p+q-\beta p q\right)^{2}}<0
\end{aligned}
$$


In the derivative above, the right-hand side is negative because factors $\beta(1-\alpha), p$, and $q$ are all positive and bounded by 1 . This property of $t^{(0)}$ is not surprising. Recall that $\mu$ is the normalized detection rate in the operating area. For a larger $\mu$, it is reasonable to expect that the target will be detected sooner.

Observation 2: $t^{(0)}$ is a decreasing function of $p$ and a decreasing function of $q$.

We differentiate $t^{(0)}$, respectively, with respect to $p$ and $q$ and obtain that

$$
\begin{aligned}
& \frac{\mathrm{d} t^{(0)}}{\mathrm{d} p}=-\frac{\left(\frac{\mu}{1-\alpha}+p+q-\beta p q\right)+(1-\beta q)\left(\frac{\mu}{\alpha}-p-q+\frac{1}{\beta \alpha(1-\alpha)}\right)}{\left(\frac{\mu}{1-\alpha}+p+q-\beta p q\right)^{2}}<0 \\
& \frac{\mathrm{d} t^{(0)}}{\mathrm{d} q}=-\frac{\left(\frac{\mu}{1-\alpha}+p+q-\beta p q\right)+(1-\beta p)\left(\frac{\mu}{\alpha}-p-q+\frac{1}{\beta \alpha(1-\alpha)}\right)}{\left(\frac{\mu}{1-\alpha}+p+q-\beta p q\right)^{2}}<0
\end{aligned}
$$

Both $\mathrm{d} t^{(0)} / \mathrm{d} p$ and $\mathrm{d} t^{(0)} / \mathrm{d} q$ are negative, which follows directly from the fact that all terms in the numerators are positive. In particular, it is true that

$$
\begin{gathered}
\frac{\mu}{1-\alpha}+p+q-\beta p q>p+q-p q>0 \\
\frac{\mu}{\alpha}-p-q+\frac{1}{\beta \alpha(1-\alpha)}>\frac{1}{\alpha(1-\alpha)}-(p+q)>4-(p+q)>0
\end{gathered}
$$

This property of $t^{(0)}$ is also expected. Remember that $p$ and $q$ are, respectively, the probability of the target being detected on its way to the operating area and the probability of being detected on its way back to the hiding area. Increasing either $p$ or $q$ while keeping the other unchanged will speed up the detection of the target.

Observation 3: While $(p+q)$ keeps fixed, $t^{(0)}$ becomes an increasing function of $(p q)$.

In the expression of $t^{(0)}$, while the sum $(p+q)$ is fixed, the combination $(p q)$ appears only in the denominator.

$$
t^{(0)}=\frac{\frac{\mu}{\alpha}-p-q+\frac{1}{\beta \alpha(1-\alpha)}}{\frac{\mu}{1-\alpha}+p+q-\beta p q}
$$

When the product $(p q)$ is increased, the denominator is decreased, and as a result, $t^{(0)}$ is increased. This behavior tells us that when evaluating several options with the same value of $(p+q)$, the optimal option is the one with the smallest value of $(p q)$.

Observation 4: $t^{(0)}$ is a decreasing function of $\beta$.

We differentiate $t^{(0)}$ with respect to $\beta$ to get

$$
\begin{gathered}
\frac{\mathrm{d} t^{(0)}}{\mathrm{d} \beta}=-\frac{\frac{\mu}{1-\alpha}\left(1-\beta^{2}(1-\alpha)^{2} p q\right)+\beta(p+q-2 p q)}{\beta^{2} \alpha(1-\alpha)\left(\frac{\mu}{1-\alpha}+p+q-\beta p q\right)^{2}} \\
-\frac{(p+q)\left(1-\beta+\beta^{2} \alpha(1-\alpha) p q\right)}{\beta^{2} \alpha(1-\alpha)\left(\frac{\mu}{1-\alpha}+p+q-\beta p q\right)^{2}}<0
\end{gathered}
$$

This property of $t^{(0)}$ is expected. $\beta$ is the fraction of time that the sensors are on. Increasing the value of $\beta$ leads to an increase in the probability that the sensors are on at a random time. and thus reduces the time to detection. 
Observation 5: When $p=q=0, t^{(0)}$ is a decreasing function of $\alpha$.

When sensors are deployed only to search in the operating area and no sensor is used to monitor any of the routes, we have $p=q=0$. In this case, $t^{(0)}$ has the simple expression

$$
t^{(0)}=\frac{1}{\alpha}\left(1+\frac{1}{\beta \mu}\right)-1
$$

which is a decreasing function of $\alpha$. When only the operating area is searched, the target can only be detected in the operating area; the target cannot be detected on its travels between the two areas. Consequently, the detection probability increases with the fraction of time that the target spends in the operating area, which is given by $\alpha$.

Observation 6: In general, $t^{(0)}$ is not necessarily a decreasing function of $\alpha$.

When the condition $p=q=0$ is not satisfied, $t^{(0)}$ may not be monotonic with respect to variable $\alpha$. Figure 8 shows a plot of $t^{(0)}$ vs $\alpha$ while other parameters are fixed at $\beta=0.55, p=0.45, q=0.23$, and $\mu=0.14$. $t^{(0)}$ initially decreases as $\alpha$ is increased from 0 . It attains a minimum around $\alpha=0.6$. For $\alpha>0.6, t^{(0)}$ appears to be an increasing function of $\alpha$. It is clear that in general, $t^{(0)}$ is not monotonic with respect to $\alpha$.

Next we study how the mean time to detection changes with $\varepsilon$, the normalized time scale of sensors relaxing to equilibrium between the on- and off-states. For that purpose, we examine coefficient $t^{(1)}$ in the asymptotic expansion $E(T)=t^{(0)}+\varepsilon t^{(1)}+\cdots$.

Observation 7: $t^{(1)}$ is always positive.

We write $t^{(1)}$ as

$$
t^{(1)}=\frac{h}{\alpha(1-\alpha)\left(\frac{\mu}{1-\alpha}+p+q-\beta p q\right)^{2}} \cdot \frac{(1-\beta)}{\beta}
$$

where $h$ is a function of $(\alpha, \beta, p, q, \mu)$, given by

$$
h=(\mu+p-\alpha(p+q))(\mu+q-\alpha(p+q))+\alpha(1-\alpha)\left(\frac{\mu}{1-\alpha}+p+q-\beta p q\right)^{2}
$$

To prove that $t^{(1)}$ is always positive, we only need to show $h>0$ is always true.

As a first step, we establish that $h$ is an increasing function of $\mu$, which is reached by differentiating $h$ with respect to $\mu$.

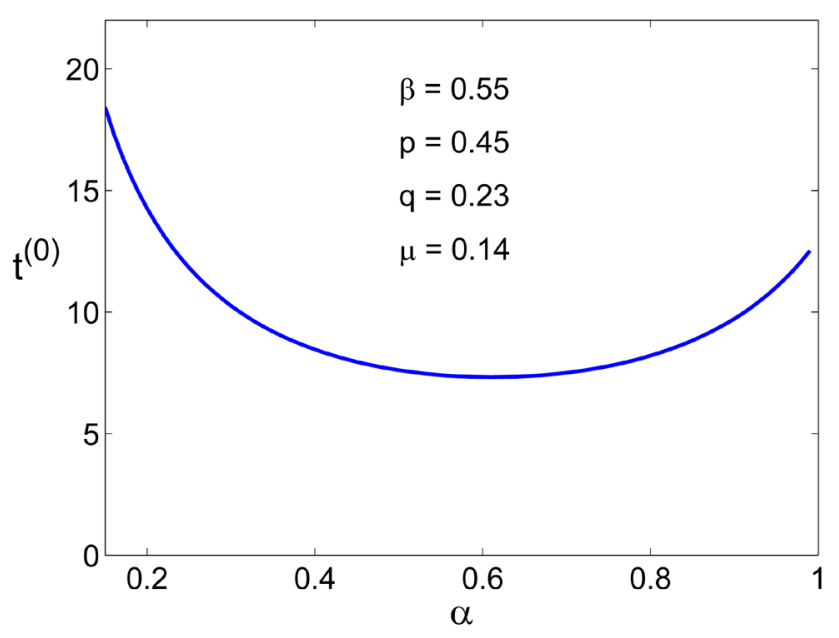

Figure 8. Plot of $t^{(0)}$ vs $\alpha$ for $\beta=0.55, p=0.45, q=0.23$, and $\mu=0.14$. Note that in general, $t^{(0)}$ is not monotonic with respect to $\alpha$. 


$$
\frac{\mathrm{d} h}{\mathrm{~d} \mu}=\frac{2 \mu}{1-\alpha}+(p+q)-2 \alpha \beta p q>(p+q)-2 p q>0
$$

Thus, to prove $h>0$, we only need to show $\left.h\right|_{\mu=0}>0$. This can be verified directly:

$$
\begin{aligned}
\left.h\right|_{\mu=0} & =(p-\alpha(p+q))(q-\alpha(p+q))+\alpha(1-\alpha)(p+q-\beta p q)^{2}>0 \\
& =p q\left(1-2 \alpha(1-\alpha) \beta(p+q)+\alpha(1-\alpha) \beta^{2} p q\right)>0
\end{aligned}
$$

In the above, we have used the facts

$$
\alpha(1-\alpha) \leq \frac{1}{4} \text { and }(p+q)<2
$$

Therefore, we conclude that $t^{(1)}$ is always positive. It follows that as a function of $\varepsilon$ while other parameters are fixed, the mean time to detection $E(T)$ decreases as $\varepsilon$ is reduced. In other words, when the fraction of time that sensors are on is fixed (i.e., $\beta$ is kept at a constant), and when the normalized time scale of sensors relaxing to equilibrium between the on- and off-states is reduced (i.e., $\varepsilon$ is made smaller), the detection performance of sensors is improved (i.e., the mean time to detection is shorter).

Finally, we demonstrate the accuracy of the asymptotic solution for the mean time to detection. Figure 9 compares an accurate numerical solution and the asymptotic expansion $t^{(0)}+\varepsilon t^{(1)}$ for $E(T)$, in the range of $\varepsilon \in[0,0.3]$. It is clear that the dependence of $E(T)$ on $\varepsilon$ is well captured in the asymptotic solution $t^{(0)}+\varepsilon t^{(1)}$ even at $\varepsilon=0.3$.

\section{Conclusion}

We have addressed the performance of stochastically intermittent sensors when used to detect a target that moves between a hiding area and an operating area via multiple routes. We have derived asymptotic expansions for the mean time to detection when the on-rate and off-rate of the sensors are large in comparison with the rates of the target moving between the hiding area and the operating area. Using the mean time to detection, we have evaluated the performance of placing sensor(s) to monitor various travel route(s) or to scan the operating area.

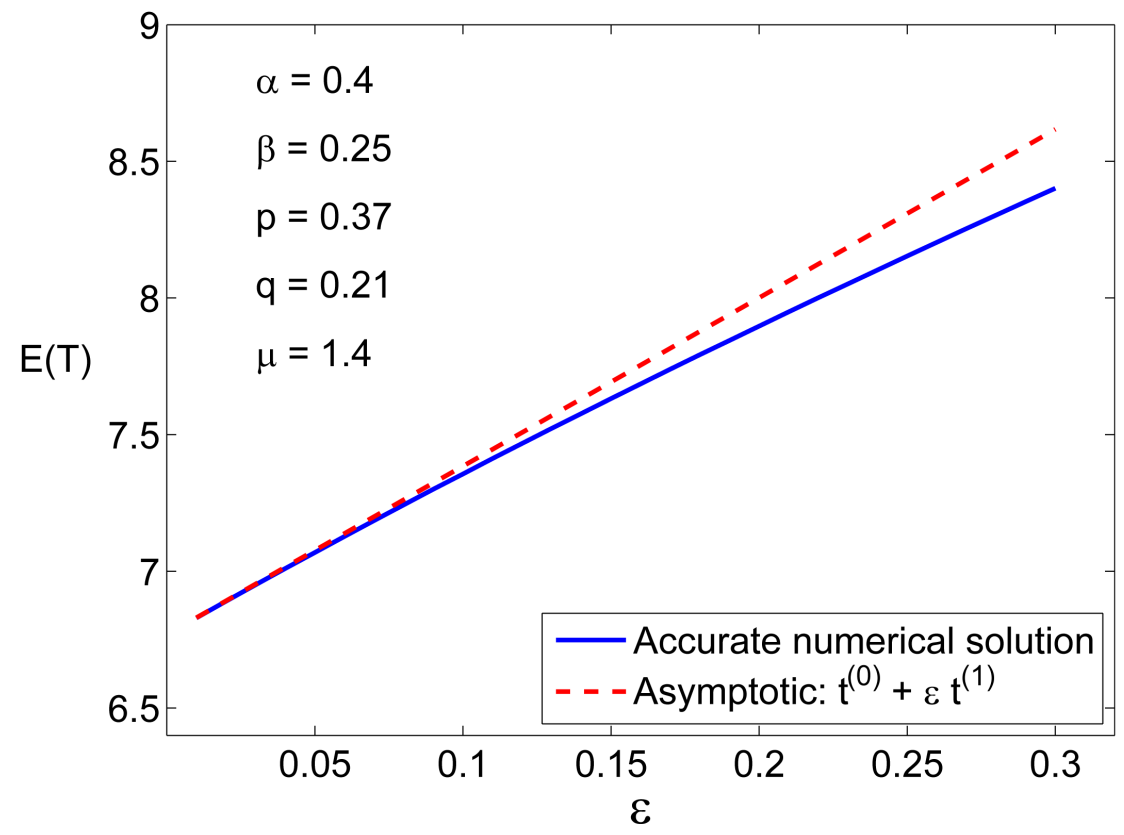

Figure 9. Comparison of an accurate numerical solution of $E(T)$ and the asymptotic solution $t^{(0)}+\varepsilon t^{(1)}$ for $\alpha=0.4, \quad \beta=0.25, \quad p=0.37, q=0.21$, and $\mu=1.4$. 


\section{Acknowledgements and Disclaimer}

Hong Zhou would like to thank Naval Postgraduate School Center for Multi-INT Studies for supporting this work. Special thanks go to Professor Jim Scrofani and Deborah Shifflett. The authors also thank Mr. Ed Waltz and Dr. Will Williamson for their inspirational suggestions. The views expressed in this document are those of the authors and do not reflect the official policy or position of the Department of Defense or the U.S. Government.

\section{References}

[1] Koopman, B.O. (1999) Search and Screening: General Principles with Historical Applications. The Military Operations Research Society, Inc., Alexandria, VA.

[2] Stone, L.D. (1989) Theory of Optimal Search. 2nd Edition, Operations Research Society of America, Arlington, VA.

[3] Wagner, D.H., Mylander, W.C. and Sanders, T.J. (1999) Naval Operations Analysis. 3rd Edition, Naval Institute Press, Annapolis, MD.

[4] Washburn, A. (2014) Search and Detection. 5th Edition, CreateSpace Independent Publishing Platforms, Location.

[5] Wang, H. and Zhou, H. (2015) Searching for a Target Traveling between a Hiding Area and an Operation Area over Multiple Routes. American Journal of Operations Research, 5, 258-273. http://dx.doi.org/10.4236/ajor.2015.54020 